\title{
Effets d'une ration à base de la variété de maïs « Espoir » sur la productivité des poulettes
}

\author{
O.C. Hien ${ }^{1 *}$, B. Diarra ${ }^{2}$ et Y. Coulibaly ${ }^{2}$ \\ ${ }^{1}$ INERA, Farako-Bâ, 01 BP 910 Bobo-Dioulasso 01, Burkina Faso. \\ ${ }^{2} U P B$, IDR 01 BP 1091, Bobo-Dioulasso 01, Burkina Faso. \\ *Auteur correspondant; E-mail: hien_ollo@yahoo.fr
}

\section{RESUME}

Au Burkina Faso, le facteur limitant dans l'alimentation des volailles est la protéine. Une étude a été menée en vue d'évaluer les effets d'une ration contenant la variété de maïs jaune «Espoir» sur les performances de croissance et de ponte chez les poulettes. Une ration contenant la variété de maïs Espoir (ESPOIR) et une deuxième contenant une variété de maïs blanc local (SR21) ont été comparées à une troisième qui était un aliment commercial contenant du maïs tout venant (CPAVI). Quatre-vingt-dix (90) poulettes Isa-Brown âgées de 11 semaines ont été aléatoirement reparties à raison de 3 lots de 10 poulettes par ration. La ration contenant le maïs Espoir a amélioré, mais pas de façon significative les paramètres mesurés sur l'ensemble de la période d'expérimentation. Les poids moyens des poules à l'entrée en ponte étaient de 1351,$0 ; 1280,8$ et $1281,0 \mathrm{~g}$ respectivement pour la ration ESPOIR, SR21 et CPAVI. En fin d'expérience, les poids moyens étaient de 1566,$1 ; 1485,9$ et 1506,0 g respectivement pour ESPOIR, SR21 et CPAVI. Le gain moyen quotidien était de 10,$0 ; 9,0$ et 9,0 g respectivement pour ESPOIR, SR21 et CPAVI. Le poids moyen des œufs à la $29^{\text {eme }}$ semaine d'âge était de 56,$6 ; 54,1$ et 55,0 g respectivement pour ESPOIR, SR21 et CPAVI. Les pics de ponte étaient de 78,6; 65,7; 67,1\% respectivement pour ESPOIR, SR21 et CPAVI. Les indices de conversion au niveau de la ponte étaient de 2,$1 ; 2,2$ et 2,2 respectivement pour ESPOIR, SR21et CPAVI. Par contre, la couleur du jaune d'œuf a été améliorée par Espoir mais demeurait blanchâtre avec CPAVI et intermédiaire avec SR21. La variété de maïs Espoir serait une alternative intéressante pour la productivité des volailles.

(C) 2017 International Formulae Group. All rights reserved.

Mots clés : Poulette, pondeuse, maïs Espoir, croissance, œuf.

\section{Effects of "Espoir" variety of corn on chicken productivity}

\begin{abstract}
In Burkina Faso, the limiting factor in poultry feed is protein. This study has been conducted to evaluate the effect of one ration containing the ESPOIR variety of a yellow corn on growth and laying performance of chicken hens. One ration containing ESPOIR and a second one containing SR21 variety of white corn (SR21) were compared to a commercial ration containing other varieties of white corns (CPAVI). ESPOIR improved but not significantly the measured parameters. Weights at $1^{\text {st }}$ egg were $1351,0,1280,0$ and $1281,0 \mathrm{~g}$ respectively for ESPOIR, SR21 and CPAVI. Mean weights at the end of the experiment were 1566,1; 1485,9, and 1506,0 g respectively for ESPOIR, SR21 and CPAVI. Average daily gains were 10,0;9,0 and 9,0g respectively for ESPOIR, SR21 and CPAVI. Mean egg weights were 56,6; 54,1 and 55,0 g respectively for ESPOIR, SR21 and CPAVI. Peak laying rates were 78,6;65,7, and 67,1\% respectively for ESPOIR, SR21 and CPAVI. Feed conversion ratios during laying were 2,1;2,2 and 2,2 respectively for ESPOIR, SR21 and CPAVI. However, the color of egg yolk turned into intense orange while remaining white yellowish for
\end{abstract}


CPAVI, and intermediary for SR21. The ESPOIR variety of corn could be an interesting alternative for a good productivity of chicken.

(C) 2017 International Formulae Group. All rights reserved.

Keywords: Pullets, laying hen, Espoir corn, growth, egg.

\section{INTRODUCTION}

Au Burkina Faso, 95\% des volailles produites proviennent de l'aviculture traditionnelle. Cette activité est facilement accessible aux ménages ruraux qui la pratiquent pour satisfaire leurs besoins et s'acquitter de leurs obligations sociales (MRA, 2007). Elle occupe environ $80,0 \%$ de la population et contribue pour $83,4 \%$ au revenu des ménages et pour $19,0 \%$ aux revenus générés par le sous-secteur de l'élevage (Ouedraogo, 2003 ; MRA, 2009).

Cependant, l'insuffisance alimentaire freine le développement de l'aviculture. Cette carence est plus marquée au niveau des protéines d'origine animale. Les farines de poissons, en plus de leurs coûts relativement élevés, peuvent être sources d'infection par les anaérobies sulfito-réducteurs, les coliformes thermo tolérants et les salmonelles (Farina et al., 1991).

Les variétés de maïs jaune augmentent l'intensité de la couleur du jaune d'œuf, un facteur important chez les consommateurs; elles sont préférées en raison de leur taux légèrement plus élevé en xanthophylle pour la coloration du jaune d'œuf (Mpouok, 1999) et en protéines riches en acides aminés essentiels (lysine et tryptophane) facilement assimilables par l'homme. Deux rations alimentaires pour lapin incorporant d'une part la variété de maïs jaune Espoir et d'autre part la variété de maïs blanc SR21 ont révélé un taux de protéines plus élevé dans la ration contenant le maïs Espoir (Ouattara, 2012). Le maïs Espoir pourrait alors constituer une alternative pour réduire l'apport en protéines animales et entraîner ainsi une diminution du coût de production. C'est dans cette optique que la variété de maïs «Espoir» est étudiée pour évaluer les performances de croissance et de ponte chez les poules.

\section{MATERIEL ET METHODES Site d'étude}

L'étude a été conduite à la station de recherche de Farako-Bâ, située à $10 \mathrm{~km}$ de la ville de Bobo-Dioulasso (longitude $4^{\circ} 20^{\prime}$ Ouest, latitude $11^{\circ} 06^{\prime}$ Nord et altitude $405 \mathrm{~m}$ ). Le climat est de type soudano guinéen avec une saison sèche allant de novembre à mai et une saison pluvieuse de mai à octobre; la pluviométrie moyenne annuelle est comprise entre 900 et $1200 \mathrm{~mm}$ (Guinko, 1984).

\section{Le poulailler}

Il s'agit d'un bâtiment de $5,5 \mathrm{~m}$ sur 4,5 m qui a été aménagé en 9 boxes de 2,24 $\mathrm{m}^{2}$ chacun. Les boxes étaient faits en grillage avec une hauteur de $1,5 \mathrm{~m}$ et contenaient chacun une mangeoire linéaire de $1 \mathrm{~m}$ et un abreuvoir automatique de 61 et 10 poules. La toiture faite en tôle avait une hauteur de $7 \mathrm{~m}$. La ventilation du bâtiment était naturelle avec une orientation Nord-Sud. La litière, faite de balles de riz recouvrait le sol et était renouvelée chaque mois. L'élevage a été conduit sous photopériode naturelle.

\section{Le matériel animal et alimentation}

Le matériel animal était constitué de 90 poulettes ISA-Brown rouge âgées de 11 semaines. La provende utilisée au cours de l'expérience (Tableau 1) était fournie par le Centre de Promotion de l'Aviculture Villageoise (CPAVI), qui est un atelier de fabrique d'aliment commercial de bétail. Les deux variétés de maïs «ESPOIR » et «SR21 » ont été fournies au CPAVI par les producteurs de maïs encadrés par la station de recherche de Farako-Bâ.

Les poulettes, d'un poids moyen de $716,1 \pm 15,5 \mathrm{~g}$, ont été choisies au hasard parmi un lot de 500 poulettes. L'expérience comportait 3 traitements comprenant chacun 3 lots de 10 poulettes. L'étude a consisté à comparer trois variétés différentes de maïs, Espoir, SR21 et du maïs tout venant (CPAVI) dans des rations des aliments commerciaux contenant le même taux de chacune des 3 variétés. Les quantités d'aliments refusées étaient pesées et relevées quotidiennement dans le but de connaître la consommation journalière. Le suivi de la croissance des 
poulettes a duré 10 semaines. A 20 semaines d'âge, les poules ont donné leur $1^{\text {er }}$ œuf; la ponte a été suivie pendant 11 semaines. Les œufs étaient collectés chaque matin et pesés.

\section{Prophylaxie vétérinaire et sanitaire}

L'hypochlorite de sodium (eau de javel) a été utilisé pour désinfecter le poulailler juste avant le transfert des poulettes. Les vaccins contre le Gumboro, la variole et le New Castle ont été administrés lors de l'expérience. Les antistress, anti-infectieux, anticoccidiens, antibiotiques et les complexes vitaminés ont aussi été appliqués dans l'eau de boisson.

\section{Paramètres mesurés}

Les paramètres mesurés pendant les périodes de croissance étaient: l'ingestion quotidienne d'aliment, les poids hebdomadaires, le gain moyen quotidien (GMQ) et l'indice de consommation (I.C). Cependant, pendant la période de ponte, il s'agissait de mesurer l'indice de consommation (IC) par œuf, le taux de ponte, le poids des œufs, la qualité de l'œuf, la coloration du jaune d'œuf et le taux de mortalité.

\section{Analyses statistiques}

Le dispositif utilisé est le plan des blocs complètement randomisés $3 \times 3$. Les graphiques ont été effectués par le logiciel Excel 2010. Le logiciel SAS/STAT, 2010 (version 9.2) a été utilisé pour les traitements statistiques. L'analyse de variance a été effectuée par la méthode ANOVA. La séparation des moyennes a été effectuée par la méthode de Tukey au seuil de 5\%.

Tableau 1 : Formule alimentaire des rations CPAVI pour volaille.

\begin{tabular}{lcc}
\hline Ingrédients & Poulettes (\% de ration) & Pondeuses (\% de ration) \\
\hline Maïs (Espoir, SR21 ou CPAVI) & 56,37 & 57,80 \\
Son de moulin & 14,46 & 14,50 \\
Son de blé & 14,45 & 7,24 \\
Tourteau de coton & 6,93 & 6,37 \\
Tourteau de soja & 0,71 & - \\
Poisson & 2,05 & 7,05 \\
Coquille & 3,37 & 5,9 \\
Sel & 0,14 & 0,14 \\
Pré-mix & 0,25 & 0,25 \\
Lysine & 0,28 & 0,24 \\
Méthionine & 0,08 & 0,06 \\
Phosphate bicalcique & 0,85 & 0,58 \\
Sulfate de fer & 0,11 & 0,12 \\
\hline TOTAL & $\mathbf{1 0 0}$ & $\mathbf{1 0 0}$ \\
\hline
\end{tabular}




\section{RESULTATS}

Phase croissance

Analyse calculée des rations pour poulettes

Le Tableau 2 présente l'analyse calculée des rations pour poulettes. Les taux de protéines obtenus avec les variétés de maïs SR21 et ESPOIR étaient plus élevés mais pas de façon significative par rapport au maïs CPAVI $(8,3$ à $11,1 \%)$ qui comprenait à la fois des variétés de maïs blanc et jaunes. Les valeurs moyennes étaient de 9,1, 11,2 et $11,47 \%$ respectivement pour le maïs CPAVI, SR21 et ESPOIR.

Performances des poulettes de 12 à 20 semaines

Consommation moyenne d'aliment

Durant toute la période de croissance (Tableau 3), la consommation moyenne quotidienne d'aliment par poule n'avait pas varié de façon sensible $(\mathrm{P}>0,05)$; elle était respectivement de $73,7,71,5$ et $70,5 \mathrm{~g}$ pour ESPOIR, SR21 et CPAVI.

Poids moyen, GMQ et IC des poulettes en fin de croissance

Les moyennes des poids, des GMQ et des IC n'ont pas connu de différences significatives $(\mathrm{P}>0,05)$ entre les trois traitements. Pour les 3 traitements, les poids des poulettes ont augmenté régulièrement et similairement de la $12^{\text {ère }}$ semaine jusqu'à l'entrée en ponte à la $20^{\text {ème }}$ semaine (Figure 2) ; Les moyennes des GMQ et des l'IC étaient centrées dans les intervalles respectives de 7,7 $\pm 1,2 \mathrm{~g}$. Aucune mortalité n'a été observée pendant la phase croissance.

\section{Phase ponte}

Analyse des rations pour pondeuses

Le Tableau 4 présente l'analyse calculée des rations pour pondeuses. Les taux de protéines des rations-ponte étaient aussi similaires, de $15,1 \%$ pour CPAVI et augmentant seulement de 7,9 et $8,6 \%$ respectivement pour SR21 et ESPOIR.

\section{Poids moyen des pondeuses}

Il n'y a pas eu non plus de mortalité au cours de la ponte. En fin d'expérience, le poids moyen des poules n'était pas différent. Les valeurs étaient centrées dans l'intervalle 1519,3 \pm 80,7 g (Tableau 4).

\section{Consommation moyenne d'aliments}

Les ingestions alimentaires des trois traitements n'ont montré aucune différence $(\mathrm{P}$ $>0,05)$, les valeurs par pondeuse étaient de
105,9 \pm 9,6 g. Elles étaient en général ascendante de la $1^{\text {ère }}$ semaine de ponte jusqu'à la fin de l'expérience et avaient tendance à se confondre à partir de la $7^{\text {ème }}$ semaine (Figure $1)$.

Nombre moyen d'oufs et Taux de ponte

L'entrée en ponte a été observée précocement, dès la $19^{\text {ème }}$ semaine pour ESPOIR et SR21 mais pratiquement deux semaines plus tard pour CPAVI.

Le nombre moyen d'œufs obtenu pendant toute la période expérimentale était environ 30,0\% plus élevé avec ESPOIR, mais numériquement seulement $(\mathrm{P}>0,05), 33,6 \%$ contre $26,2 \%$ et $25,5 \%$ respectivement pour SR21 et CPAVI. Le nombre a augmenté progressivement pour les trois traitements jusqu'à la $7^{\text {ème }}$ semaine correspondant au pic de ponte et baissé légèrement à partir de cette date. Corolairement, les pics sont aussi apparus tôt, dès la $25^{\text {ème }}$ semaine d'âge.

Poids moyen des aufs

Le poids moyen des œufs n'a pas connu de différences significatives $(\mathrm{P}>0,05)$ pour les trois traitements, même si avec Espoir, les œufs étaient $2 \mathrm{~g}$ plus lourds durant les 2 premières semaines (Figure 3). Les poids étaient centrées dans l'intervalle 49,0 \pm $2,9 \mathrm{~g}$.

\section{Indice de conversion}

Les IC des trois traitements se situent dans la marge de 2-2,4 comme rapportée par ITAVI (2002). Les valeurs étaient similaires $(\mathrm{P}>0,05)$ dans l'intervalle étroit de 2,1 $\pm 0,2$.

\section{Diamètre du jaune d'auf}

Dix œufs sélectionnés au hasard au niveau de chaque traitement ont été cassés et leurs diamètres mesurés. Le diamètre moyen obtenu avec ESPOIR était plus large de façon non significative $(P>0,05)$, de seulement 0,1 $\mathrm{cm}, 3,7$ vs. $3,6 \mathrm{~cm}$ pour SR21 et CPAVI.

\section{Couleur du vitellus}

L'évaluation de la couleur du jaune du vitellus a été faite de façon visuelle sur une échelle de 100. La différence entre les traitements était nette. Pour le traitement SR21 utilisant du maïs blanc local, le vitellus était de couleur blanc jaunâtre (5\%). Comparativement à SR21 l'intensité de la couleur jaune obtenu avec CPAVI était beaucoup plus intense $(80 \%)$ et encore plus intense avec ESPOIR (95\%), prenant une couleur orange intense. 
Tableau 2: Analyse calculée des rations pour poulettes.

\begin{tabular}{llccc}
\hline & $\begin{array}{l}\text { Energie métabolisable } \\
\text { Kcal/kg }\end{array}$ & $\begin{array}{c}\text { Protéines } \\
\text { (\% de } \mathbf{M S})\end{array}$ & $\begin{array}{c}\text { Ca } \\
\text { (\% de MS) }\end{array}$ & $\begin{array}{c}\text { P } \\
\text { (\% de MS) }\end{array}$ \\
\hline CPAVI & 2783,6 & 13,7 & 1,9 & 0,6 \\
SR21 & 2783,6 & 14,8 & 1,9 & 0,6 \\
ESPOIR & 2783,6 & 14,8 & 1,9 & 0,6 \\
\hline
\end{tabular}

Tableau 3: Effets des traitements sur les performances des poulettes de 12 à 20 semaines.

\begin{tabular}{lcccc}
\hline Paramètres & ESPOIR & SR21 & CPAVI & Moyenne générale \\
\hline Poids moyen à 12 semaines $(\mathrm{g})$ & $1051,6 \mathrm{a}$ & $1012,9 \mathrm{a}$ & $1022,0 \mathrm{a}$ & $1028,2 \pm 188,7$ \\
Poids moyen à l'entrée en ponte $(\mathrm{g})$ & $1351,0 \mathrm{a}$ & $1280,8 \mathrm{a}$ & $1281,0 \mathrm{a}$ & $1304,2 \pm 204,7$ \\
Aliment consommé par poule, $\mathrm{g} / \mathrm{j}$ & $73,7 \mathrm{a}$ & $71,5 \mathrm{a}$ & $70,5 \mathrm{a}$ & $71,9 \pm 11,3$ \\
GMQ, $\mathrm{g}$ & $10,0 \mathrm{a}$ & $9,0 \mathrm{a}$ & $9,0 \mathrm{a}$ & $9,3 \pm 1,5$ \\
IC & $7,4 \mathrm{a}$ & $7,9 \mathrm{a}$ & $7,8 \mathrm{a}$ & $7,7 \pm 1,2$ \\
Mortalité, \% & $0,0 \mathrm{a}$ & $0,0 \mathrm{a}$ & $0,0 \mathrm{a}$ & $0,0 \pm 0,0$ \\
\hline \multicolumn{2}{c}{ Les valeurs qui portent la même lettre sur la même ligne ne sont pas significativement différentes au seuil de 5\%. }
\end{tabular}

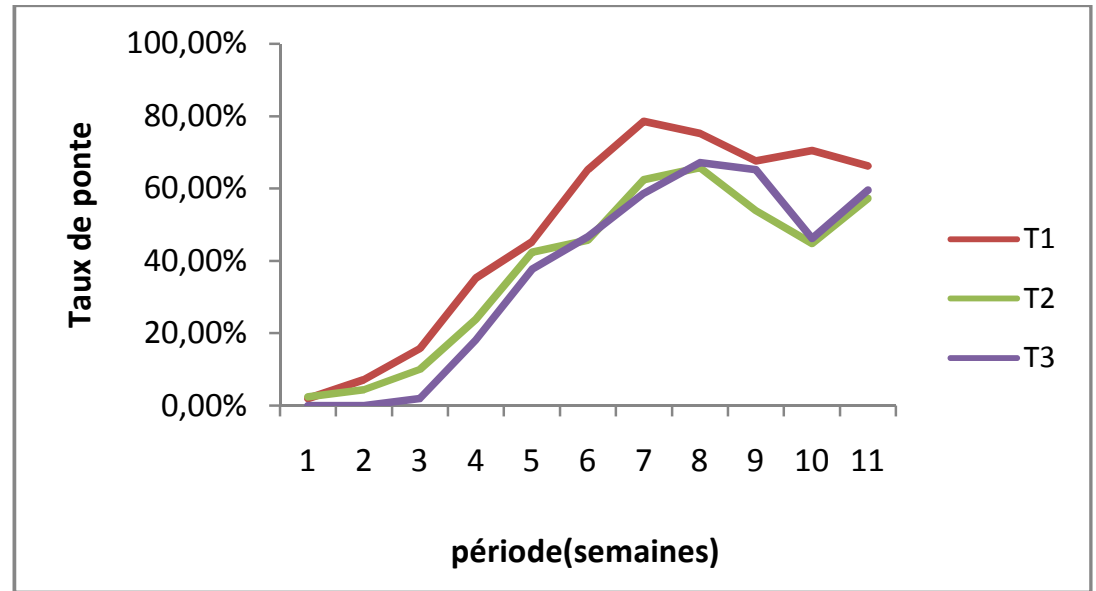

Figure 2 : Evolution du taux de ponte par traitement. T1 : ESPOIR, T2 : SR21, T3 : CPAVI 
Tableau 4 : Analyse calculée des rations pour pondeuses

\begin{tabular}{lllll}
\hline & Energie métabolisable & Protéines & Ca & P \\
\hline CPAVI & 2790,6 & 15,1 & 3,1 & 0,7 \\
SR21 & 2790,6 & 16,3 & 3,1 & 0,7 \\
ESPOIR & 2790,6 & 16,4 & 3,1 & 0,7 \\
\hline
\end{tabular}

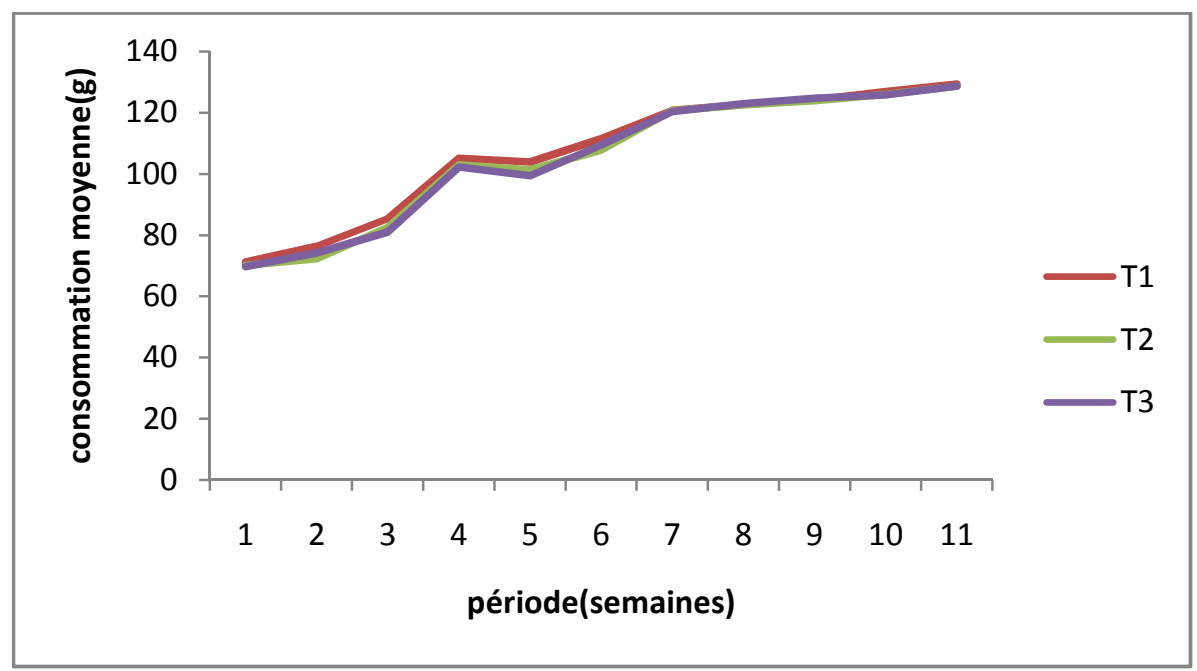

Figure 1 : Evolution de la consommation moyenne d'aliment. T1: ESPOIR, T2: SR21, T3: CPAVI 


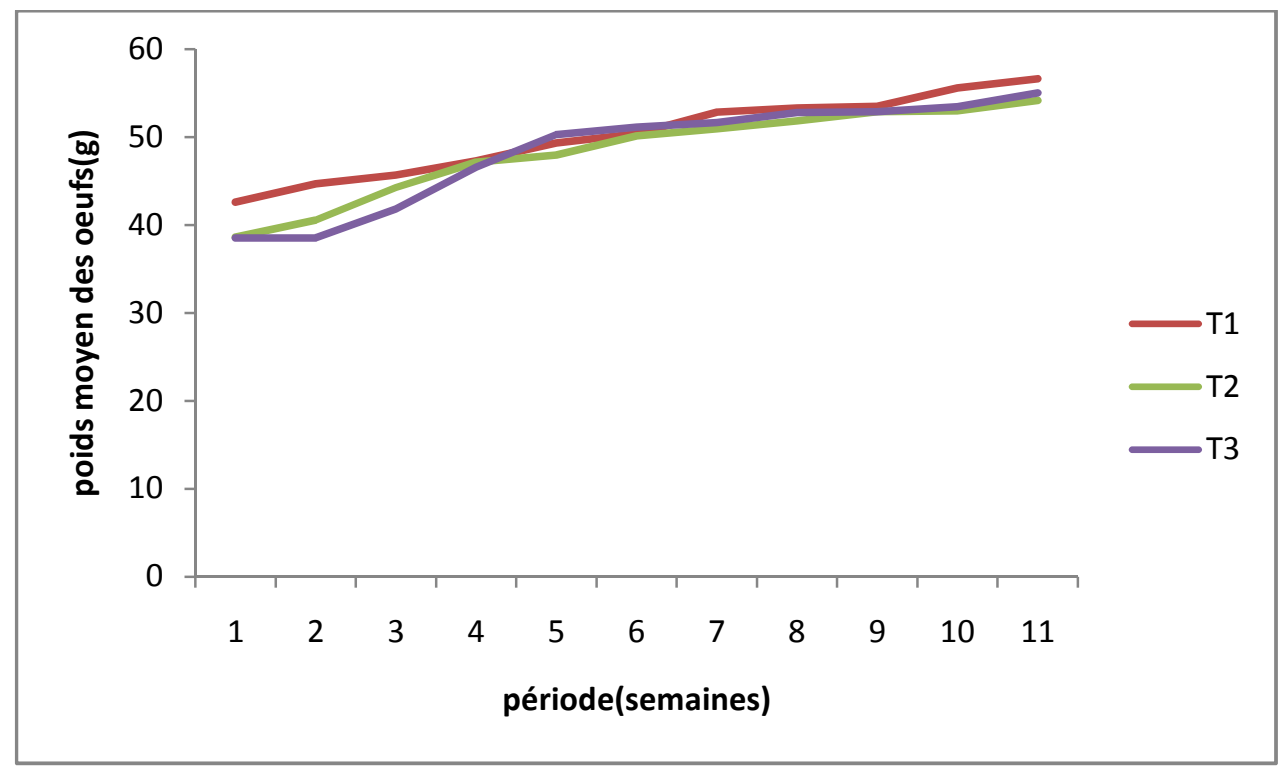

Figure 3 : Evolution du poids moyen des œufs par traitement. T1: ESPOIR, T2: SR21, T3 : CPAVI.

Tableau 5 : Valeurs moyennes des paramètres zootechniques des pondeuses de 19 à 29 semaines d'âge.

\begin{tabular}{lcccc}
\hline Paramètres & ESPOIR & SR21 & CPAVI & Moyenne générale \\
\hline Consommation d'aliments par poule, g & $107,0 \mathrm{a}$ & $105,3 \mathrm{a}$ & $105,2 \mathrm{a}$ & $105,9 \pm 9,6$ \\
Poids moyen à la $29^{\text {eme }}$ semaine d'âge, $\mathrm{g}$ & $1566,1 \mathrm{a}$ & $1485,9 \mathrm{a}$ & $1506,0 \mathrm{a}$ & $1519,3 \pm 80,7$ \\
Indice de conversion & $2,1 \mathrm{a}$ & $2,2 \mathrm{a}$ & $2,1 \mathrm{a}$ & $2,1 \pm 0,2$ \\
Nombre moyen d'œufs par poule & $33,6 \mathrm{a}$ & $26,2 \mathrm{a}$ & $25,5 \mathrm{a}$ & $28,5 \pm 9,8$ \\
Poids moyen de l'œuf, $\mathrm{g}$ & $50,2 \mathrm{a}$ & $48,3 \mathrm{a}$ & $48,4 \mathrm{a}$ & $49,0 \pm 2,9$ \\
Diamètre du jaune d'œuf, cm & $3,7 \mathrm{a}$ & $3,6 \mathrm{a}$ & $3,6 \mathrm{a}$ & $3,6 \pm 0,1$ \\
Taux de mortalité, \% & $0,0 \mathrm{a}$ & $0,0 \mathrm{a}$ & $0,0 \mathrm{a}$ & \\
\hline
\end{tabular}




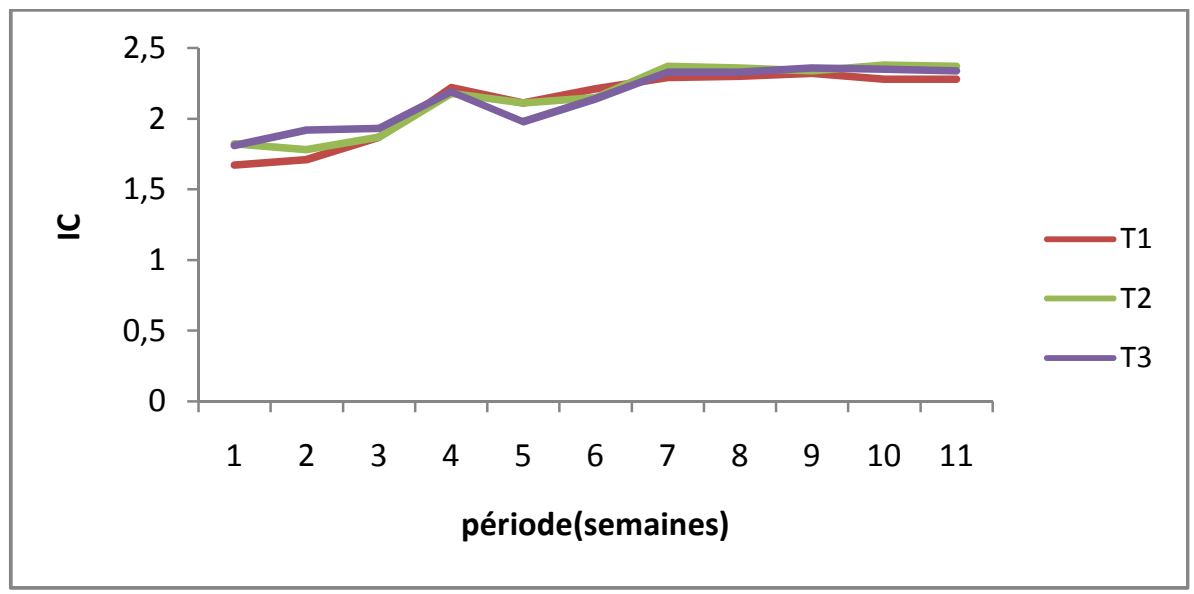

Figure 4 : Evolution de l'indice de consommation.

\section{DISCUSSION}

De l'analyse calculée des rations poulettes

En considérant que les taux d'énergies métabolisables, de calcium et de phosphore restent constants, seul le taux de protéines constaté avec CPAVI $(13,7)$ a augmenté mais de façon non significative, seulement de 8,0 et $8,8 \%$ respectivement par rapport à SR21 et ESPOIR (Tableau 2). Conséquemment, les augmentations de taux de protéines espérées avec ces nouvelles variétés se sont montrées aussi faibles dans les rations des poulettes, augmentant de 1 point.

Des performances des poulettes de 12 à 20 semaines Consommation moyenne d'aliment

Hancock et al. (2007) avait également conclu que la source de la céréale et la taille des particules n'avaient aucun effet significatif sur l'ingestion alimentaire, mais il s'agissait de la substitution du grain de maïs par celui du sorgho qui sont tous deux des grains de céréales. Cependant, à l'exception des grains de céréales, Nworgu et Fasoghon (2007) ont rapporté que Cetrosema pubescens utilisé comme source de protéines avait augmenté la consommation alimentaire des poulettes Black Nera âgées de 10 semaines; tel était le cas aussi avec des feuilles de Moringa oléifera introduites à $10 \%$ dans la ration des poulettes white Leghorn de 21 semaines d'âge (Kakengi et al., 2007)

Poids moyen, GMQ et IC des poulettes en fin de croissance

Les poids moyens à la $12^{\text {ème }}$ semaine étaient centrés dans les intervalles 1028,2 \pm 188,7 , correspondant à l'intervalle de 1010 à $1050 \mathrm{~g}$ du standard d'élevage de ISA Brown. L'entrée en ponte a été observée à la $20^{\text {ème }}$ semaine comme rapporté dans le standard d'élevage d'ISA Brown (ISA, 2010). Cependant, le poids d'entrée en ponte de 1304 $\pm 204,7 \mathrm{~g}$ est inférieur de $18,9 \%$ au $1550 \mathrm{~g}$ préconisés pour la $18^{\text {ème }}$ semaine d'âge dans le standard d'élevage. Avec le maïs Espoir, le poids d'entrée en ponte était légèrement plus élevé, de $70 \mathrm{~g}$, par rapport à SR21 et CPAVI qui avaient des valeurs très proches. Ceci exprime que la variété Espoir a une plus grande efficacité de valeur nutritive parce qu'elle recèle un taux de protéines plus élevé et pourrait être un avantage pour une bonne croissance des poulettes. Cela concorde avec 
les conclusions de Kondombo (2000) et de Nougtara (2011) qui ont relevé que les protéines constituent le principal facteur limitant pour la croissance des oiseaux. Aucune mortalité n'ayant été observée pendant la phase croissance indique que les poulettes ont été conduites dans des conditions sanitaires acceptables et alimentaires équilibrées.

\section{Phase ponte}

\section{Poids moyen des pondeuses}

Les valeurs qui étaient centrées dans l'intervalle 1519,3 \pm 80,7 g (Tableau 4) étaient 22,4\% moins lourds que les $1860 \mathrm{~g}$ rapportés pour ISA Brown à la $29^{\mathrm{ème}}$ semaine.

\section{Consommation moyenne d'aliments}

Les valeurs par pondeuse qui étaient de $105,9 \pm 9,6 \mathrm{~g}$ étaient $7.6 \%$ inférieur à l'ingestion de $114 \mathrm{~g}$ rapportées pour ISA Brown. L'ingestion volontaire des pondeuses ISA Brown a été située dans l'ordre de $105 \pm$ $15 \mathrm{~g}$ dans plusieurs expériences (Agunbiade et al., 2007 ; Kakengi et al., 2007 ; Houndonougbo et al., 2012). Les besoins nutritifs augmentant avec la ponte, amène les pondeuses à consommer plus afin d'avoir l'énergie nécessaire pour pondre (Bronkhorst, 2000). La production d'œufs entraîne un besoin accru en nourriture et selon Smith (1992), $1 \mathrm{~g}$ d'œuf produit nécessiterait $1 \mathrm{~g}$ de nourriture.

\section{Nombre moyen d'oufs et Taux de ponte}

La période de fraîcheur de décembre à janvier correspondant au début de l'allongement de la période diurne, où les poules consomment beaucoup plus d'énergie, entraînerait une ponte précoce. En saison de période lumineuse courte, les poules ont les taux de ponte les plus faibles. Notre expérience couvrait le mois de novembre où la durée de la période lumineuse est la plus courte dans l'année, inférieure à 12 heures (Hien, 2002). Aussi, sans lumière artificielle, les taux de ponte étaient dans l'ensemble faibles, inférieurs à 40,0\% jusqu'à la fin du premier mois.

Cependant, dans les expériences de Kakengi et al. (2007) et de Houndonougbo et al. (2012), des poules de 21 semaines d'âge avaient dépassé ce seuil dans le même délai ; cela serait dû au fait que les poules de nôtre étude étaient entrées en ponte deux semaines plus tôt.

Le pic ponte de $94,7 \%$ rapporté par ISA (2010) pour ISA Brown n'était pas atteint; cependant les taux de ponte étaient toujours plus élevés avec ESPOIR (Figure 2), cela de façon significative au moment du pic de ponte, $78,6 \%$ contre $65,7 \%$ pour SR21 et $67.1 \%$ pour CPAVI. Ceux obtenus par Kakengi et al. (2007) et de Houndonougbo et al. (2012), étaient constamment au-dessus de $70 \%$ durant les $2^{\text {ème }}$ et $3^{\text {ème }}$ mois.

Une carence en acides aminés essentiels limite la production d'œufs (Larbier et Leclercq, 1991). Le taux de protéines conseiller pour une ponte maximum de ISA Brown est de 18\%. La variété de maïs Espoir contenant plus de protéines contiendrait aussi plus d'acides aminés essentiels (lysine et tryptophane) et de vitamine A que les deux autres variétés.

\section{Poids moyen des oeufs}

Bien que le poids des œufs soit fonction de l'âge et du poids des poules, la qualité de l'aliment distribué y contribue (Sauveur, 1988). En effet, les protéines font partie des éléments nutritionnels qui influencent la ponte et le poids des œufs est fonction des protéines qui y sont contenues (Sauveur, 1988). Un abaissement du taux protidique alimentaire va entraîner une réduction du poids de l'œuf portant davantage sur le blanc (Sauveur, 1988). Le poids moyen de l'œuf est réduit significativement sous l'effet d'une déficience en méthionine (Leclercq, 1970). Dans notre étude, le poids moyen des œufs aux dernières semaines (55 à $58 \mathrm{~g})$ se rapproche de celui rapporté par (Gallot, 2006) qui se situe entre 52 et $60 \mathrm{~g}$ et inférieur à celui enregistré par (ITAVI, 2002) qui est compris entre 58 et $60 \mathrm{~g}$.

\section{Le diamètre et la couleur du jaune d'ouf}

Bien qu'aucune différence significative n'existe entre les traitements pour le diamètre des jaunes d'œufs le maïs Espoir offrait un jaune d'œuf légèrement volumineux par rapport aux deux autres traitements qui étaient identiques. Ceci est en accord avec (Sauveur, 1988) qui affirmait que plus l'œuf est lourd, plus le jaune est volumineux. 
La couleur du jaune d'œuf a montré une différence entre les traitements à travers les photos. Ainsi il ressort que la coloration $\mathrm{du}$ jaune d'œuf est fonction de la couleur du maïs utilisé dans la ration. Les maïs jaunes sont riches en xanthophylles colorant le jaune de l'œuf en jaune (Diallo, 1981). Les poules sont incapables de synthétiser les caroténoïdes, mais les accumulent très facilement dans le jaune de l'œuf. Leur alimentation contient des pigments (présents dans les matières premières ou ajoutés) qui permettent d'ajuster la coloration du jaune d'œuf à la demande des consommateurs. Seules les xanthophylles (caroténoïdes présentant un groupement oxygène) ont un effet colorant (Sauveur et Nysi, 2000). N'étant pas capables de les synthétiser, les volailles trouvent ces pigments rouges et jaunes dans leur alimentation sous forme de xanthophylles (Siri, 2007). Les œufs au jaune d'œuf jaune sont les plus prisés par les consommateurs, parce qu'ils apportent en plus des protéines, des vitamines. Les volailles locales (poules, pintades) qui divaguent et qui ont le loisir de choisir leurs aliments pondent des œufs au jaune de coloration jaune, au goût succulent.

\section{Conclusion}

La substitution du maïs blanc SR21 par la variété de maïs jaune Espoir a amélioré mais de façon non significative le poids des poulettes, le taux de ponte, le poids moyen des œufs et l'indice de conversion, mais de façon significative la coloration du jaune d'œuf, ce qui pourrait contribuer à améliorer davantage la qualité organoleptique des œufs à la grande satisfaction des consommateurs.

La variété de maïs Espoir représente une perspective intéressante pour améliorer l'alimentation des poules au Burkina Faso.

\section{CONFLIT D'INTERETS}

Les auteurs déclarent qu'il n'y a aucun conflit d'intérêts pour cet article.

\section{REFERENCES}

Agunbiade JA, Adeyemi OA, Ashiru OM, Awojobi HA, Taiwo AA, Oke DB, Adekunmisi AA. 2007. Replacement of Fish meal with Maggot meal in cassava- based layers 'diets'. The Journal of Poultry Science, 44: 278-282.

Diallo H. 1981. Contribution à l'étude de l'alimentation des volailles en HauteVolta. Mémoire du diplôme d'ingénieur du Développement Rural, IDR/UPB, 124 P.

Gallot S. 2006. Evolution de la production et de la consommation de volailles et d'œufs de consommation au niveau mondial. Service Economie ITAVI, 24 p.

Hancock J. 2007. Sorghum: A substitute for maize in Nicaraguan Poultry Industry, INTSORMIL Report $N^{\circ}$ 13, April 1, 2007, 2 p.

Hasin BM, Ferdaus AJM, Islam MA, Uddin MJ, Islam MS. 2006. Morigold and orange skin as egg yolk colour promoting agents. International Journal of Poultry Science, 5(10) : 979-987.

Hien OC. 2002. Effets de l'amélioration des conditions sanitaires sur les performances de reproduction de la pintade locale du Burkina Faso, thèse, Université de Ouagadougou, BF, 130 p.

Houndonougbo MF, Chrysostome CAAM, Houndonougbo VP. 2012. Performances bioéconomiques des poulettes alimentées avec des rations à base de feuilles séchées de manioc (Manhiot esculenta). Int. J. Biol. Chem. Sci., 6(2): 670-676.

Houndonougbo MF, Chrysostome CAAM, Houndonougbo VP. 2012. Performances de ponte et qualité des œufs des poules pondeuses ISA Brown alimentées avec des rations à base de feuilles séchées de manioc (Manhiot esculenta). Int. J. Biol. Chem. Sci., 6(5):1950-1959.

ITAVI, AFSSA, CIRAD. 2002. La Production d'Eufs en Climat Chaud. Ed. ITAVI, $120 \mathrm{p}$.

ISA. A Hendrix Genetics Company. 2010. Conduite technique des élevages de poulettes et de pondeuses en climat chaud, $17 \mathrm{p}$.

Kakengi AMV, Kaijage JT, Sarwatt SV, Mutayoba SK, Shem MN, Fujiyama T. 2007. Effect of Moriga oleifera leaf meal as a substitute for sunflower seed meal on performance of laying hens in 
Tanzania. Livestock Research for Rural Developmnt, 19(8). Online.

Kondombo RS. 2000. Case study on production systems and feeding of village chicken in the central region on Burkina Faso, MSc thesis, animal sciences, Wageningen University, $90 \mathrm{p}$.

Leclercq B. 1970. Facteurs nutritionnels modifiant le poids de l'œuf et de ses constituants. Ann. Biol. Anim. Bioch. BioPhys., 10: 239-252.

Larbier M, Leclerq B. 1991. Nutrition et Alimentation des Volailles. INRA, Paris, $355 \mathrm{p}$.

Mpouok O. 1999. Contribution à la mise au point d'un référentiel sur la qualité des matières premières utilisées en aviculture au Sénégal : application à la formulation des rations alimentaires, thèse de Docteur Vétérinaire, Ecole Inter-états de Science et Médecine Vétérinaire, Université Cheick Anta Diop de Dakar UCADD, $88 \mathrm{p}$.

MRA (Ministère des Ressouces Animales). 2007. Diagnostic de la sous filière de l'aviculture traditionnelle au Burkina Faso. Rapport final. $62 \mathrm{p}$.

MRA (Ministère des Ressouces Animales). 2009. Politique de développement de l'élevage du Burkina-Faso, 2010-2020. Burkina- Faso, $45 \mathrm{P}$.

Nworgu FC, Fasoghon FO. 2007. Centrosema (Centrosema pubescens) leaf meal as protein suplement for pullet chicks and growing pullets. Int. J. Biol. Chem. Sci., 6(4): 255-260.

Nougtara S. 2011. Valorisation des Aliments disponibles dans l'alimentation des poulets traditionnels au Burkina Faso. Mémoire du Diplôme d'Ingénieur du Développement Rural: IDR/UPB ,89 p.

Ouattara DAKM. 2012. Régénération de lignées extraites de la variété de maïs Espoir et test d'alimentation de lapin avec la variété Espoir ; rapport présenté en vue de l'obtention du brevet de Technicien Supérieuret d'Agriculture (BTS), option agronomie; CAP de Matourkou, Bobo-Dioulasso, Burkina Faso, $40 \mathrm{p}$.

SAS 2010. User's Guide. Basics. Statistical Analysis Systems Institute, Inc. Cary, NC.

Sauveur B. 1988. Reproduction des Volailles et Production d'Eufs. Edition INRA; 11-49.

Sauveur Y, Nysi B. 2000. Comprendre la formation de la coquille de l'œuf de poule. Station de Recherches Avicoles, centre de Tours INRA, 2000.

Siri FIMR. 2007. Publication scientifique. Huitièmes Journées de la Recherche Avicole. J. Appl. Poult. Res., 429- 437.

Smith AJ. 1992. L'élevage de la volaille, Premier volume. Volailles situées dans les zones chaudes. In Production de Poulets de Chair (edn 17 - 20). Maisonneuve et Laroche; ACCT, CTA : Paris ; 183.

Vervacketal W, Vanbelle M, Foulon M, Moreau I. 1983. Composition en acides aminés des œufs de ferme et des œufs de production industrielle. Revue fermentation et des industries. 